\title{
Particularités du collage aux dents qui ont subi un éclaircissement
}

\section{Specific aspects of bonding after teeth whitening}

MOTS-CLEFS :
- Éclaircissement
dentaire, collage,
force d'adhésion,
agent anti-oxydatif
KEYWORDS:
- Teeth bleaching,
bonding, bond strength,
Anti-oxidative agent

$\operatorname{AOS} n^{\circ} 287-2018$

\section{Résumé}

L'action oxydative du peroxyde d'hydrogène est responsable de la diminution de la force d'adhésion immédiatement après un éclaircissement. L'oxygène résiduel interfère avec la polymérisation et la diffusion de l'adhésif dans les anfractuosités dentaires. Pour le faire échapper, il faut reporter les procédures de collage de deux semaines en cas d'éclaircissement interne. Après un éclaircissement externe, l'emploi des agents anti-oxydatifs, tel que l'ascorbate de sodium, permet d'éviter la diminution de la force d'adhésion, permettant ainsi le collage immédiatement après l'éclaircissement. L'éclaircissement à concentration élevée peut modifier les propriétés mécaniques et esthétiques des restaurations adhésives préexistantes, d'où la nécessité de les remplacer après un traitement d'éclaircissement afin d'optimiser le résultat esthétique final.

\begin{abstract}
The oxidative action of hydrogen peroxide is responsible for the decrease in bond strength immediately after bleaching treatment. The residual oxygen interferes with the polymerization and the diffusion of the adhesive in dental anfractuosities. A delay of bonding for two weeks after an intra-coronal bleaching can reverse this effect. After an external bleaching, an anti-oxidative agent, such as sodium ascorbate, can avoid the decrease in bond strength, thus, bonding process can be performed immediately after bleaching...

The mechanical and aesthetic properties of pre-existing adhesive restorations are altered after Bleaching using high concentrations. They need to be replaced after a whitening treatment in order to optimize the final aesthetic result.
\end{abstract}

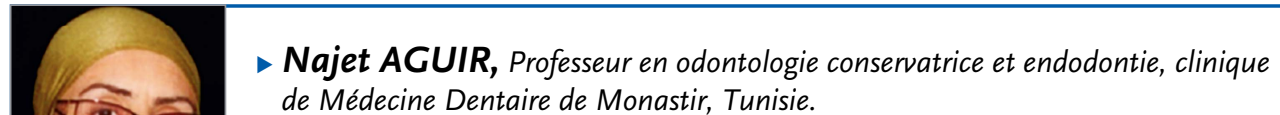
de Médecine Dentaire de Monastir, Tunisie.

Mayada JEMÂA, Assistante Hospitalo-Universitaire, service de Médecine et Chirurgie Dentaire, Hôpital Militaire Principal d'Instruction de Tunis, Tunisie.

Emna JALOULI, Médecin Dentiste, Tunisie.

MB. KHATTECH, Professeur en Médecine et Chirurgie Buccale, service de Médecine et Chirurgie Dentaire, Hôpital Militaire Principal d'Instruction de Tunis, Tunisie.

Lotfi BHOURI, Professeur en odontologie conservatrice et endodontie, clinique de Médecine Dentaire de Monastir, Tunisie. 


\section{INTRODUCTION}

Actuellement, la demande esthétique est devenue une véritable préoccupation dans notre société de plus en plus marquée par les médias. En effet, cette société est en quête continue de dents d'une blancheur éclatante et de sourires attractifs. Un sourire attractif est synonyme de bonne santé, beauté, réussite et intégration sociale.

L'éclaircissement dentaire, vital et non vital, est une thérapeutique qui trouve parfaitement sa place au sein de l'arsenal des traitements esthétiques proposés. L'éclaircissement dentaire n'est pas sans effets sur certaines procédures thérapeutiques ultérieures telles que le collage des restaurations adhésives. En effet, l'action oxydative du peroxyde d'hydrogène, utilisé en éclaircissement dentaire, peut interférer avec la formation d'une couche hybride de bonne qualité en réduisant la force d'adhésion des restaurations adhésives aux tissus dentaires.

Différents auteurs ont étudié l'interface dent/résine composite et analysé les divers facteurs pouvant influencer le collage aux dents éclaircies.

Le but de notre article est d'analyser à travers une revue de la littérature :

I L'impact de l'éclaircissement sur la procédure de collage aux tissus dentaires éclaircis.

\ Les facteurs à l'origine de la diminution de la force d'adhésion des restaurations adhésives après un éclaircissement dentaire.

\Les effets de l'éclaircissement sur les propriétés des restaurations adhésives préexistantes et la nécessité de les remplacer.

I Les recommandations et les astuces qu'il faut suivre pour optimiser la qualité du collage après un éclaircissement dentaire.

\section{EFFETS DE L'ÉCLAIRCISSEMENT VITAL SUR LE COLLAGE AUX STRUCTURES DENTAIRES RÉSIDUELLES}

Les agents de blanchiment ont été étudiés à des concentrations variables pour évaluer les effets du blanchiment sur la force d'adhésion des restaurations adhésives.

Les résultats de ces études ont montré une réduction significative de la force d'adhésion des résines composites à l'émail blanchi et l'absence de liaison entre les ciments verres ionomères modifiés par adjonction de résine et cet émail immédiatement après un éclaircissement.

Danesh-Sani et coll. (2011) ont montré que l'éclaircissement vital avec le peroxyde d'hydrogène à $9.5 \%$ (6 heures par jour durant 7 jours) réduit significativement la résistance au cisaillement et par conséquence la force d'adhésion de la résine composite (Z100,3M/ ESPE). Il ne permet pas non plus le collage du CVI MAR (Vitremer, 3M/ESPE) aux structures dentaires. (Tableau 1) Il semble que la liaison ionique entre le groupe carboxylique de l'acide polyalkenoique et l'hydroxyapatite a été totalement inhibée par l'oxygène résiduel de la réaction d'oxydation du produit de l'éclaircissement [1].

Gurgan et coll. (2009) ont montré que l'éclaircissement vital avec le peroxyde de carbamide à $16 \%$ et à $30 \%$ entraîne une diminution de la résistance au cisaillement de la résine composite nano-hybride collée à l'émail et à la dentine sans qu'il y ait une différence significative entre les deux concentrations $(\mathrm{p}>0.05)[2]$.

\section{EFFETS DE L'ÉCLAIRCISSEMENT NON VITAL SUR LE COLLAGE AUX STRUCTURES DENTAIRES RÉSIDUELLES}

Différentes études ont été réalisées dans le but d'évaluer la qualité du collage suite à un éclaircissement interne. Ces dernières ont mis en garde contre des collages imparfaits aux structures dentaires à l'origine de l'augmentation des microinfiltrations à l'interface des restaurations collées et de la diminution de la force d'adhésion de la résine composite collée immédiatement après un éclaircissement non vital $[3,4,5]$.

Rahimi et coll. (2010) ont constaté que l'éclaircissement non vital au peroxyde d'hydrogène à $30 \%$ a augmenté significativement la micro-infiltration à l'interface adhésive avec la résine composite (Z100, 3M/ESPE) (Tableau 2) [4]. 


\begin{tabular}{|c|c|c|c|}
\hline Groupes & \multicolumn{4}{|c|}{ Degrés de la micro-infiltration } \\
\hline A & 0 & I & II \\
\hline B & 5 & 7 & 3 \\
\hline
\end{tabular}

Degré 0 : pas de pénétration du colorant ;

Degré I : pénétration du colorant dans l'émail ;

Degré II : pénétration du colorant dans l'émail et la dentine ;

(A) Groupe non éclairci et restauré par le Z100, 3M/ESPE ;

(B) Groupe éclairci et restauré par le Z100.3M/ESPE (Peroxyde

d'hydrogène à $30 \%$ ).

$\triangle$ Tableau 2: Effet de l'éclaircissement non vital au peroxyde d'hydrogène à $30 \%$ sur les restaurations par la résine composite Z100, 3M/ESPE.

Prabhakar et coll. (2010) ont montré une diminution statistiquement non significative de la résistance au cisaillement des restaurations à la résine composite des dents éclaircies par rapport à celles des dents témoins juste après un éclaircissement interne au peroxyde de carbamide à $10 \%$ et $22 \%$ [6].

Cependant, Shinohora et coll. (2004/2005) ont montré que l'éclaircissement non vital pendant 7 jours, avec le perborate de sodium affecte défavorablement la force d'adhésion de la résine composite à l'émail et à la dentine et le peroxyde de carbamide à $37 \%$ affecte l'adhésion à l'émail mais pas à la dentine $[7,8]$.

Feiz et coll. (2011) ont constaté que l'éclaircissement interne par le peroxyde d'hydrogène à $35 \%$ a entravé l'adhésion immédiate de la résine composite (Z100, 3M/ESPE) aux tissus dentaires. [9]

\section{FACTEURS INFLUENÇANT L'ADHÉSION AUX TISSUS DENTAIRES ÉCLAIRCIS}

Différentes études ont conclu que la diminution de la force d'adhésion est essentiellement due à l'oxygène résiduel de l'agent de l'éclaircissement. Il inhibe partiellement la polymérisation de la résine et réduit son infiltration dans les tissus dentaires mordancés [2, 4, $10,11,12,13]$.

La photo-polymérisation est sévèrement ralentie par les molécules d'oxygène, présentes dans l'air et de l'oxygène résiduel du produit éclaircissant. La réaction

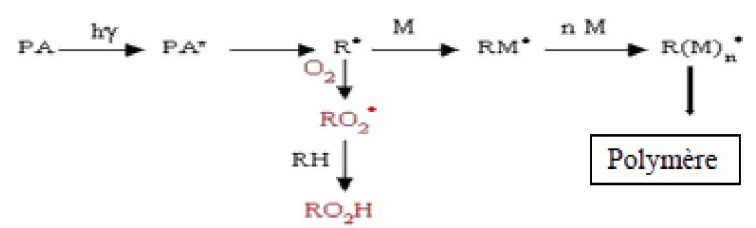

$\triangle$ Figure 1 : Réaction de $\mathrm{l}^{\prime} \mathrm{O}_{2}$ avec les radicaux libres inhibant par endroit la polymérisation [14]. de polymérisation radicalaire est inhibée à cause de la forte réactivité de l'oxygène moléculaire vis-à-vis des radicaux libres $(\mathrm{R} \bullet)$. L'oxygène réagit avec les radicaux engendrés par le photo-initiateur pour produire des peroxydes ( $\mathrm{RO} 2 \bullet)$ et des hydro-peroxydes $(\mathrm{RO} 2 \mathrm{H})$, qui occupent les anfractuosités de l'émail et les tubuli dentinaires, interrompent par endroit la polymérisation, affectent l'hybridation tissulaire et par conséquence l'étanchéité de l'interface dent éclaircie/ résine [14] (Figure 1).

Le peroxyde résiduel occupe les anfractuosités de l'émail et les tubuli dentinaires qui constituent la clé de l'adhésion mécanique par micro clavetage. Il se décompose de temps en temps en oxygène et en eau pouvant arrêter la diffusion de l'agent de collage dans ces rugosités.

D’autres études ont suggéré que la déminéralisation des tissus dentaires, la diminution de leur énergie de surface et la dénaturation du collagène dentinaire suite à l'éclaircissement peuvent également expliquer cette réduction de la force d'adhésion $[4,10,15,16]$.

L'analyse des images de l'interface résine/émail éclairci par microscope électronique a montré des brides résineuses moins abondantes, fragmentées, plus courtes et de pénétration moins profonde par rapport au groupe contrôle non éclairci. La qualité et la quantité des prolongements résineux sont en relation directe avec la valeur de la force d'adhésion $[4,10,17,18,19$, 20] (Figure 2 et 3 ).

\section{EFFETS DE L'ÉCLAIRCISSEMENT SUR LES TISSUS DENTAIRES}

Plusieurs auteurs ont suggéré qu'un éclaircissement est à l'origine de modifications de la structure de l'émail. L'augmentation de sa porosité qui se mani-
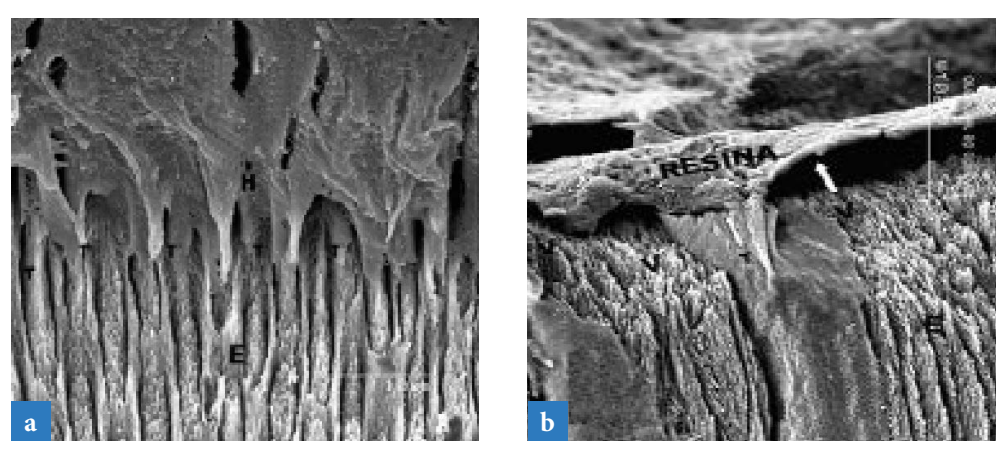

$\triangle$ Figure 2 : Images par MEB à fort grossissement de l'interface émail/résine montrant la qualité et la quantité des brides résineuses dans les anfractuosités de l'émail non éclairci (a) et l'émail éclairci (b) x 5000 [20]. (a) : l'interface émail non éclairci/résine est constituée d'une couche hybride avec des multiples brides résineuses ( $\mathrm{T}$ : tags) bien définis et pénètrent profondément dans les anfractuosités de l'émail mordancé (E). (b) : l'interface émail éclairci/résine est constituée de prolongements résineux rares et fragmentés $(T)$, avec absence de couche hybride et d'imprégnation, par endroit $(\mathrm{V}, \mathrm{V})$, de la résine avec l'émail (E). 

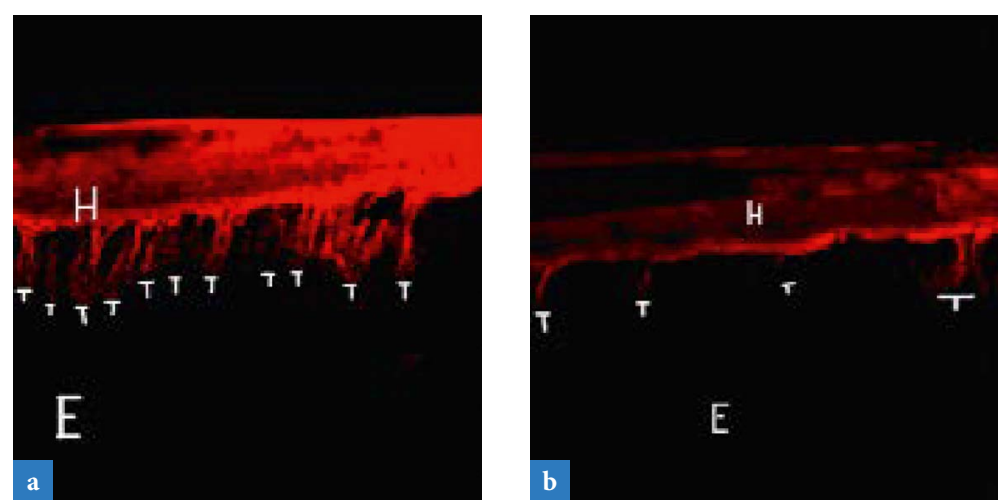

$\triangle$ Figure 3 : Images par microscopie confocal par fluorescence de l'interface résine/émail du groupe témoin non éclairci (a) et du groupe collé 48 heures après éclaircissement (b) [20]. $(\mathrm{H})$ couche hybride, $(\mathrm{T})$ prolongements résineux, (E) émail. (a) : l'adhésif pénètre profondément les anfractuosités de l'émail non éclairci donnant des brides de résine bien définies. (b) : réduction significative de la pénétration de l'adhésif dans les microporosités de l'émail éclairci qui se traduit par des brides résineuses rares courtes et indéfinies.

feste par la perte de sa forme prismatique, la perte des ions calcium, phosphore, sulfure et potassium, les changements du rapport $\mathrm{Ca} / \mathrm{P}$ et la réduction de sa micro-dureté sont autant de facteurs participant à la diminution de la force d'adhésion des restaurations collées [16, 21, 22, 23, 24].

Tam et al. (2007) ont conclu la réduction de la résistance à la fracture de la dentine lorsqu'elle est directement exposée au peroxyde de carbamide à $10 \%$ pour une durée d'application 6 heures par jour et pendant $5 \mathrm{j} /$ semaine durant 5 semaines [25].

Chang et al (2005) ont démontré la réduction significative de la microdureté et du module de Young de la dentine inter-tubulaire après utilisation du peroxyde d'hydrogène à $30 \%$ pour une durée d'application de 24 heures [26].

En résumé, le $\mathrm{pH}$ acide, les applications répétées et prolongées et les concentrations élevées des produits d'éclaircissement peuvent contribuer à la déminéralisation des tissus durs dentaires par libération ionique causant une diminution de leur micro-dureté et une augmentation de leurs rugosités de surface $[21,23,26,27]$.

La couche hybride est un entrelacement de deux types de polymères: les fibres de collagène de la matrice dentinaire, polymère d'origine naturelle, d'une part, et les macromolécules de l'adhésif, polymère de synthèse d'autre part. Sous l'effet des radicaux libres de l'oxygène résiduel, il se produit une dénaturation du collagène, diminuant leur imprégnation par la résine et la force d'adhésion de celle-ci aux structures dentinaires. La qualité de la couche hybride ainsi que l'étanchéité de la restauration adhésive ne seront plus optimales [28]. La force d'adhésion aux tissus dentaires est liée à leur énergie de surface qui conditionne la mouillabilité ou le mouillage et l'étalement de l'adhésif amélo-dentinaire sur le substrat dentaire. La mouillabilité représente l'aptitude d'un liquide à occuper la plus grande surface tissulaire possible. La tension superficielle ou l'énergie de surface des adhésifs utilisés doit être inférieure à celle des tissus à coller pour permettre une mouillabilité optimale et un maximum de liaisons entre l'adhésif et le substrat amélo-dentinaire [29].

La réaction d'oxydation de l'éclaircissement réduit l'énergie de surface des tissus dentaires et affecte ainsi leur mouillabilité par des agents de collage hydrophobes et donc leur adhésion [10].

Un traitement de surface préalable au collage est pratiquement indispensable pour augmenter l'énergie superficielle du substrat. Ce traitement doit avoir comme but principal de nettoyer les tissus dentaires de tous les éléments susceptibles d'opposer une barrière à l'établissement des liaisons [29].

\section{OPTIMISATION DE L'ADHÉSION AUX STRUCTURES DENTAIRES ÉCLAIRCIES}

Différents facteurs modifiant la qualité du collage des restaurations adhésives aux tissus dentaires éclaircis ont incité plusieurs auteurs à tester l'efficacité d'une diversité de traitements afin d'éviter la réduction de la force d'adhésion. D'autres études comparatives ont été menées afin de déterminer le moyen le plus efficace.

\section{Le délai d'attente}

Uysal et al. (2003) ont conservé les échantillons éclaircis par le peroxyde d'hydrogène à $35 \%$ dans la salive artificielle pendant 30 jours avant de procéder au collage. Ils ont suggéré qu'un délai de 2 à 3 semaines avant le collage est suffisant pour une régénération complète des forces d'adhésion aux tissus dentaires éclaircis [30].

Barbosa et coll. (2008) ont recommandé un délai de 7 jours entre la fin d'éclaircissement par le peroxyde d'hydrogène à $35 \%$ et le collage sur l'émail, et de 14 jours pour le collage sur la dentine [32].

Usual et coll. (2009) ont remarqué que l'immersion des échantillons éclaircis au peroxyde de carbamide à $16 \%$ dans la salive artificielle pendant 30 jours avant le collage a augmenté la force d'adhésion mais sans atteindre la valeur du groupe témoin [12].

Kilinç et al. (2016), ont suggéré qu'après éclaircissement interne le collage à l'émail est recommandé après 14 jours ou après 7 jours suite à l'application de l'ascorbate de sodium [31].

Reporter le collage après un éclaircissement permet de faire échapper l'oxygène résiduel et de régénérer les propriétés adhésives originales des tissus dentaires résiduels. 
Plusieurs auteurs ont suggéré d'autres alternatives pour neutraliser les effets des produits d'éclaircissement et permettre ainsi les obturations adhésives immédiatement après un éclaircissement.

Ils ont mis l'accent sur l'apport de certains anti oxydants dans la suppression des radicaux libres et la neutralisation de l'oxygène résiduel.

\section{Traitement antioxydatif \\ Les antioxydants enzymatiques}

Les trois enzymes qui constituent la base du système anti-oxydatif de défense sont : la dismutase de superoxyde, la catalase et la peroxydase de glutathion.

Pour contrôler le peroxyde d'hydrogène, le corps humain utilise deux enzymes : la catalase et la peroxydase de glutathion [33].

\section{La peroxydase de glutathion}

La peroxydase de glutathion fournit deux hydrogènes (H-) qui réagissent avec le peroxyde d'hydrogène pour donner deux molécules d'eau [53].

\section{La catalase}

La catalase est formée de quatre chaines polypeptidiques d'environ 500 acides aminés, comportant chacune un groupe hème. Pour catalyser la réaction, l'atome de fer du groupement hème de la catalase réalise une coupure hétéro-lytique de la liaison $\mathrm{O}-\mathrm{O}$ du peroxyde d'hydrogène, créant de ce fait une molécule d'eau et un groupement Fe (IV) = O hautement oxydant; ce dernier peut ensuite oxyder une autre molécule de peroxyde d'hydrogène pour donner du dioxygène. Avec une vitesse maximale de 200000 réactions catalytiques par seconde, la catalase est une des enzymes les plus efficaces connues.

Carols et coll. et Einer et coll. recommandent l'application de 10 à $20 \mathrm{ml}$ de solution aqueuse de catalase sur les échantillons éclaircis pendant 5 à 20 minutes pour des concentrations de peroxyde d'hydrogène de $35-38 \%[20,33]$.

Selon Carlos (2006) et Einer (2004), l'application de la catalase donne des valeurs de force d'adhésion plus importantes que celles de la peroxydase de glutathion [20,33].

Une étude comparative (2004) évaluant l'efficacité de différents agents anti oxydants, suite à un éclaircissement externe, a montré que la catalase donne les valeurs des forces d'adhésion les plus élevées par rapport à la peroxydase de glutathion et l'ascorbate de sodium à $10 \%$ [20].

Carlos et coll. (2006) ont analysé l'effet de l'ascorbate de sodium à $10 \%$, catalase, peroxydase de glutathion, acétone, éthanol, et bicarbonate de sodium à $7 \%$ sur la neutralisation des produits résiduels de l'éclaircissement. La catalase appliquée pendant 20 minutes sur des dents éclaircies avec du peroxyde d'hydrogène à $35 \%$ semble être le traitement antioxydant le plus efficace [33].

\section{Les antioxydants non enzymatiques}

Parmi les antioxydants non enzymatiques, nous pouvons citer comme exemples : la vitamine E (les tocophérols), l'ascorbate de sodium et la béta-carotène [34].

\section{L'ascorbate de sodium}

L'ascorbate de sodium compromet le processus d'incorporation des ions peroxydes dans les structures dentaires et permet la pénétration profonde de la résine dans les anfractuosités dentaires.

Il permet par ailleurs une polymérisation complète des radicaux libres de l'adhésif et la formation d'une couche hybride étanche et uniforme à l'interface résine/dent $[34,35,36]$.

L'ascorbate de sodium est préféré à l'acide ascorbique pour éviter l'effet de double mordançage à l'A. ortho-phosphorique et à l'A. Ascorbique [35]. L'ascorbate de sodium peut être utilisé sous forme de solution ou sous forme d'hydrogel.

Une solution d'ascorbate de sodium à $10 \%$ est préparée en diluant $10 \mathrm{~g}$ de poudre cristallisée de l'ascorbate de sodium dans l'eau distillée, cette solution doit être préparée juste avant son application sur les structures dentaires [37].

Danech-Sani et coll. (2011) ont indiqué que l'application de l'ascorbate de sodium à $10 \%$ pendant 10 minutes régénère parfaitement la force d'adhésion réduite de la résine composite à l'émail, après un éclaircissement au Peroxyde d'Hydrogène à 9,5\%. Ils ont par ailleurs montré qu'il n'y a pas de différence significative entre l'effet antioxydant de l'ascorbate de sodium à $10 \%$ et l'immersion des échantillons éclaircis dans la salive artificielle pendant 7 jours avant le collage sur la force d'adhésion de la résine composite. [1].

Bhusari et coll. (2017) ont suggéré que l'application de catalase et l'ascorbate de sodium après éclaircissement améliorent la reminéralisation par la salive selon différents modèles. Et aucun de ces antioxydants ne permet de rétablir la topographie originelle de l'émail [38].

Mageshwaran et coll. (2014) ont conclu que l'ascorbate de sodium est supérieur au proanthocyanidine et au lycopene pour inverser la force d'adhésion des résines composites à l'émail éclairci [39].

Anirudh et coll. (2015) ont conclu que le traitement de la surface amélaire éclaircie par l'aloe vera et l'ascorbate de sodium à $10 \%$ permet une meilleure force de collage. L'aloe vera peut être utilisée comme une alternative à l'ascorbate de sodium à $10 \%$ [40]. 


\section{Autres alternatives \\ La fluoration}

La fluoration permet de reminéraliser les tissus durs dentaires affectés par l'éclaircissement et de réduire l'hypersensibilité [10,41].

Da Costa et coll. (2007) ont constaté que l'émail éclairci avec le peroxyde de carbamide à $10 \%$, associé au nitrate de potassium à $3 \%$ et au fluor à $0,11 \%$, a une micro-dureté similaire à celle de l'émail non éclairci [42].

Cependant, Tschoppe et coll. (2009) n'ont pas observé un effet reminéralisant significatif des gels éclaircissant contenant du fluor [43].

\section{Les adhésifs à base de solvants organiques}

La réduction de la force d'adhésion à l'émail éclairci peut également être liée à son énergie de surface.

L'émail mordancé a une énergie de surface très élevée. L'oxydation provoquée par l'agent de l'éclaircissement réduit l'énergie de surface de l'émail, cela affecterait probablement la mouillabilité de ce substrat par des agents de liaison hydrophobes.

Des études antérieures ont supposé que l'utilisation de systèmes adhésifs contenant des solvants organiques tel que l'éthanol ou l'acétone peut pallier les effets négatifs de l'éclaircissement sur le collage [10, 25]. La tension de surface réduite des adhésifs contenant des solvants organiques (alcool, acétone) peut augmenter la mouillabilité des surfaces dentaires éclaircies et donc, la pénétration de la résine dans les irrégularités crées par le mordançage et favoriser ainsi la qualité du collage [10]. Cependant, Carlos et coll. (2006) ont testé la capacité anti-oxydative de l'éthanol et de l'acétone sur l'émail. Ils ont montré qu'ils n'ont pas d'effet significatif sur les valeurs de la force d'adhésion. Cela montre leur incapacité à neutraliser les radicaux libres du peroxyde d'hydrogène [33].

Selon Niat et coll. (2012), l'emploi d'adhésif à base d'acétone améliore beaucoup plus la force de collage des résines composites à l'émail éclairci que l'adhésif à base d'alcool [44].

\section{Les Proanthocyanidines}

La Proanthocyanidine a montré des effets sur la réticulation et le renforcement du collagène dentinaire [45].

\section{Le bicarbonate de sodium}

Le bicarbonate de sodium à $7 \%$ a été indiqué par les fabricants pour neutraliser l'agent d'éclaircissement résiduel. Son $\mathrm{pH}$ basique $[8,67]$ peut déstabiliser la molécule de peroxyde et l'inactiver.

Selon Carlos et coll. (2006) le traitement de l'émail éclairci avec le bicarbonate de sodium à $7 \%$ donne une augmentation non significative de la valeur de la force d'adhésion [33].

\section{L'hydroxyde de calcium}

La réduction du $\mathrm{pH}$ au niveau de la jonction amélodentinaire occasionnée par l'agent éclaircissant fait de la résorption externe une complication courante de l'éclaircissement interne.

L'hydroxyde de calcium est connu par son $\mathrm{pH}$ basique. Son application temporaire dans la chambre pulpaire permet de neutraliser le $\mathrm{pH}$ et d'inhiber l'activité des ostéoclastes [4, 46].

Rahimi et coll. (2010) et Feiz et coll. (2011) ont constaté que l'éclaircissement non vital avec le peroxyde d'hydrogène à $30 \%$ a augmenté significativement la micro-infiltration de la résine composite (Z100, 3M/ ESPE), et que l'emploi de l'hydroxyde de calcium avant le collage ne prévient pas ces micro-infiltrations [4]. L'hydroxyde de calcium n'a, par ailleurs, aucun effet sur la force d'adhésion des restaurations adhésives aux tissus dentaires éclaircis.

\section{Le laser}

Andrias et coll. (2011) ont démontré que le conditionnement des surfaces dentaires avec le laser Er:YAG favorise le collage de la résine composite 24 heures après l'éclaircissement $[47,48,49]$.

Gurgan et coll. (2009) ont comparé l'effet sur la force d'adhésion aux dents éclaircies avec du peroxyde de carbamide à $16 \%$ et à $30 \%$ des systèmes adhésifs M\&R et systèmes adhésifs auto mordançants précédées par une application ciblée d'un faisceau de laser Er,Cr:YSGG aligné perpendiculairement à une distance de $1 \mathrm{~mm}$ de l'émail, pendant 30 secondes avec une fréquence de $20 \mathrm{~Hz}$ et une puissance de $1 \mathrm{~W}$, de $55 \%$ d'eau et $65 \%$ d'air. Ils ont conclu que la force d'adhésion des restaurations réalisées avec des systèmes adhésifs $M \& R, 15$ jours après l'éclaircissement, est plus élevée que celle obtenue avec les systèmes adhésifs auto-mordançants et l'application de laser [2].

\section{EFFETS DE L'ÉCLAIRCISSEMENT SUR LES RESTAURATIONS ADHÉSIVES PRÉEXISTANTES}

Les produits d'éclaircissement sont à l'origine d'altérations des restaurations adhésives notamment leur teinte, leur rugosité de surface et leur dureté.

Un éclaircissement au peroxyde d'hydrogène ou au peroxyde de carbamide à concentration basse (6-15\%) ne cause pas une dissolution significative du CVI [50]. La micro-dureté, l'état de surface et le coefficient d'expansion thermique de ces restaurations sont par contre affectés par les produits d'éclaircissement à concen- 
tration comprise entre 15 et $35 \%$. Elles devraient être remplacées à la fin du traitement [51].

Le compomère a la résistance la plus faible aux agents d'éclaircissement par rapport aux autres matériaux de restauration; il est recommandé de reprendre ces restaurations après la fin du traitement [52].

L'éclaircissement entraîne des changements perceptibles à l'œil nu de la couleur et de l'état de surface de ces restaurations d'où la nécessité de les remplacer après un éclaircissement $[15,50]$.

\section{RECOMMANDATIONS}

Informer le patient des modifications qui peuvent se produire au niveau des dents et des restaurations pré existantes avant de commencer le traitement de l'éclaircissement.

I Il est judicieux de choisir le produit le moins concentré possible puisqu'il engendre moins de sensibilité et moins d'effets secondaires sur la morphologie et la structure des dents et des restaurations préexistantes. I Utiliser un agent d'éclaircissement à pH neutre car un produit très acide est à l'origine de déminéralisations importantes au niveau de l'émail et donc du manque d'étanchéité des restaurations collées après un éclaircissement.

I Respecter la durée de l'exposition aux agents éclaircissants et arrêter le traitement une fois la teinte désirée est obtenue. Un éclaircissement prolongé est souvent à l'origine de dégradation des structures moléculaires de la dent.

I Procéder à des applications topiques de fluorures de sodium neutre à (1,23\%-2\%) durant 5 minutes, avant et après le blanchiment ou uniquement après le blanchiment afin de diminuer la déminéralisation et la sensibilité des tissus dentaires. Essayer des gels éclaircissants fluorés $(0,11 \%-0,5 \%)$ afin d'accélérer la régénération de la micro-dureté affectée des tissus dentaires éclaircis.

I Remplacer les restaurations antérieures en résine composite après un éclaircissement à fortes concentrations ( $16 \%$ et plus).

I Remplacer les restaurations en ciment verre ionomère et en compomère vu qu'elles sont souvent altérées.

I Appliquer l'hydroxyde de calcium après un éclaircissement intra-coronaire pendant deux semaines afin de prévenir la résorption cervicale externe. Cette durée d'application est souvent suffisante pour faire échapper l'oxygène résiduel.

I Réaliser le collage au moins 2 semaines après un éclaircissement externe ou interne.

- Appliquer une solution de catalase pendant 5 à 20 minutes avant les restaurations adhésives immé- diates. Ce traitement anti-oxydatif est le plus efficace après un éclaircissement externe.

Appliquer une solution ou un hydrogel d'ascorbate de sodium à $10 \%$ pendant $10 \mathrm{~min}(1 / 3$ la durée du traitement d'éclaircissement) pour une restauration adhésive immédiate. L'utilisation de l'ascorbate de sodium au lieu de l'acide ascorbique est recommandée pour éviter l'effet de double mordançage.

$\checkmark$ L'ascorbate de sodium est moins efficace que la catalase mais il est plus accessible comme traitement, sa préparation est aisée et moins coûteuse.

\Choisir un adhésif à base de la pro anthocyanidine permettant un potentiel de réticulation et de renforcement du collagène de la couche hybride.

\section{CONCLUSION}

Une réduction des forces d'adhésion des restaurations collées est prédictible quand le collage est réalisé immédiatement après un éclaircissement. Elle résulte essentiellement de l'oxygène résiduel dans l'émail et la dentine qui inhibe la polymérisation des résines, et interfère avec la pénétration des brides résineuses dans les anfractuosités amélaires et dentinaires. Auxiliairement de la déminéralisation des tissus durs, la diminution de l'énergie de surface dentaire et la dénaturation du collagène dentinaire par des produits d'éclaircissement très concentrés et à $\mathrm{pH}$ très acide. Les traitements anti oxydatifs les plus satisfaisants sont la catalase et l'ascorbate de sodium.

Après un éclaircissement extra-coronaire, le traitement antioxydant immédiat le plus fiable est le délai d'attente et l'ascorbate de sodium.

L'ascorbate de sodium à $10 \%$ appliqué pendant 10 minutes avant le collage, bien que moins efficace que la catalase, reste le traitement anti oxydatif immédiat le plus acceptable sur le plan clinique du point de vue temps d'application, coût, disponibilité et facilité de préparation.

Un délai d'attente de deux semaines est le traitement antioxydant le plus efficace après un éclaircissement intra-coronaire.

L'hydroxyde de calcium utilisé après éclaircissement intra-coronaire permet de prévenir la résorption cervicale externe. Sa durée d'application de deux semaines est suffisante pour faire échapper l'oxygène résiduel.

La masse de données publiées dans de nombreuses études a montré que l'éclaircissement à concentration élevée peut modifier la couleur, l'état de surface, la micro-dureté et le scellement marginal des restaurations adhésives préexistantes, d'où la nécessité de les remplacer après un traitement d'éclaircissement afin d'optimiser le résultat esthétique. 


\section{Bibliographie}

[1] Danesh-Sani SA, Esmaili M. Effect of $10 \%$ sodium ascorbate hydrogel and delayed bonding on shear bond strength of composite resin and resin-modified glass ionomer to bleached enamel. J Conserve. Dentist 2011;14(3) 241-46.

[2] Gurgan S, Alpaslan T, Kiremitci A, Cakir FY. Effect of different adhesive systems and laser treatment on shear bond stress of bleached enamel. J Dent 2009; 37: 527-34.

[3] Kimyai S, Rahimi S, Lotfi M et al. Effect of two forms of sodium ascorbate on microleakage of composite restorations immediately after bleaching. J Dent Tehran 2009; 6: 78-84.

[4] Saeed Rahimi S, Shahriar Shahi S, Soodabeh Kimyai S. Effect of calcium hydroxide dressing on microleakage of composite restorations in endodontically treated teeth subsequent to bleaching. Med Oral Patol Oral Cir Bucal. 2010; 15 (2) : 413-6.

[5] Vassalo A. Influence du blanchiment non vital sur les collages. Clinic 2001; 22: 8-13.

[6] Prabhakar AR, Sahana S, Mahantesh T, Vishwas TD. Effects of different concentrations of bleaching agent on the micro hardness and shear bond strength of restorative materials An in vitro study. J Dent Oral Hyg 2010; 2: 7-14.

[7] Shinohara MS, Peris AR, Pimenta LA, Ambrosano GM. Shear bond strength evaluation of composite resin on enamel and dentin after non vital bleaching. J Esth Rest Dent 2005; 17 (1): 22-9.

[8] Shinohara MS, Peris AR, Rodrigues JA, Pimenta LA. The effect of nonvital bleaching on the shear bond strength of composite resin using three adhesive systems. J Adhes Dent 2004; 6(3): 205-9.

[9] Khoroushi FM, Gheisarifar M. Bond Strength of Composite resin to bleached dentin: effect of using antioxidant versus buffering agent. J Dent 2011; 8(2); 61-64.

[10] Rodrigues B. The influence of bleaching agents on enamel bond strength of a composite resin . Rev Odont UNESP 2007; 36(1):77-83.

[11] Metz MJ, Cochran MA, Matis BA, Gonzalez C. Clinical evaluation of $15 \%$ carbamide peroxide on the surface microhardness and shear bond strength of human enamel. Oper Dent 2007; 32: 427-36.

[12] Uysal T, Ozgur Er, Burak Sagsen, Ayca Ustdal. Can intracoronally bleached teeth be bonded safely? Am. J. Orthod. Dentofacial Ortho. 2009; 136(5): 690-93.
[13] Cacciafesta V, Sfondrini MF. The effect of bleaching on shear bond strength of brackets bonded with a resin-modified glass ionomère Amer J Orthod Dentof Orthop 2006; 130(1): 83-6.

[14] Zeggai N. Caractérisation par gonflement de systèmes réseaux de polymères/solvantOptimisation du procédé par la méthode de surface de réponse [Mémoire de Mastère]. Université Abou Bekr Belkaïd de Tlemcen, Faculté des Sciences - Département de Chimie ; 2014.

[15] Teixeira ECN, Ritter AV, Thompson JY Leonard RH. Effect of tray-based and trayless tooth whitening systems on microhardness of enamel surface and subsurface. Amer J Dent 2004; 17: 433-436.

[16] Sahar AH. Environmental scanning Electronic Microscope study of enamel surface after using three different bleaching agents. J Am Science 2011; 7(11): 25-28.

[17] Amaral C, Jorge A, Veloso K. The effect of in-office bleaching in combination with intracoronal bleaching on enamel and dentin bond strength and dentin morphology. J Contemp Dent Pract 2008; 5: 17-24.

[18] Cavalli V, Reis AF, Giannini M. The effect of elapsed time following bleaching on enamel bond strength of resin composite. Oper Dent 2001; 26: 597-602.

[19] Kawamato K, Tsujimato T, Beher H. Effect of the hydroxyl radical and hydrogen peroxide on tooth bleaching. J Endo 2004; 30: 45-50.

[20] Villarreal Becerra E. L'effet des antioxydants sur la force d'adhésion de l'émail éclairci avec du peroxyde d'hydrogène : Les modifications immédiates structurales et morphologiques. Disponible à partir de l'url: http://tdx.cat/bitstream/handle/10803/2508/ EVB_TESIS.pdf?sequence $=1$

[21] AlSalehi S, Wood D, Hatton P. The effect of $24 \mathrm{~h}$ non-stop hydrogen peroxide concentration on bovine enamel and dentine mineral content and microhardness $J$ Dent 2007; 35: 845-50.

[22] Sun L \& al. Surface alteration of human tooth enamel subjected to acidic and neutral $30 \%$ hydrogen peroxide. J Dent 2011; 39: 686 692.

[23] Silva A, Oliviera R, Cavalli V, Arrais CAG, Giannini M. Effect of peroxidebased bleaching agents on enamel ultimate tensile strength. Oper Dent 2005; 20: 318-324.

[24] Souza RO, Lambardo GH, Pareira SM. Analysis of tooth enamel after excessive bleaching: a study using scanning electron microscopy and energy dispersive $\mathrm{x}$-ray spectroscopy. Int J Prosthodont 2010; 23: 29-32.
[25] Sung EC, Chan SM, Mito R, Caputo AA Effect of carbamide peroxide bleaching on the shear bond strength of composite to dental bonding agent enhanced enamel. J Prosthet Dent 1999; 82: 595-9.

[26] Chang HA, Ramli HN, Yapa AU, Lim CT. Effect of hydrogen peroxide on intertubular dentine. J Dent 2005; 33: 363-9.

[27] Tam L, Kuo V, Noroozi A. Effect of prolonged direct and indirect peroxide bleaching on fracture throughness of human dentin. J Esthet Restor Dent 2007; 19: 100-10.

[28] Degrange M, Pourreyron L. Les systèmes adhésifs amélo-dentinaires. 2010 disponible à partir de l'url : http://umvf.univ-nantes.fr/ odontologie/enseignement/chap12/site/html/ cours.pdf.

[29] Savoir plus sur le collage. Disponible à partir de l'url : http://materiaux.ecam.fr/ savoirplus/collage/+Collage.html

[30] Uysal T, Faruk A, Basciftci S. Previously bleached teeth be bonded safely? Amer J Orthod Orthop 2003; 123(6): 628-32.

[31] Kılınç Hİ, Aslan T, Kılıç K, Er Ö, Kurt G. Effect of delayed bonding and antioxidant application on the bond strength to enamel after internal bleaching. J Prosthodont 2016; 25(5): 386-91.

[32] Barbosa CM, Sasaki RT, Flório FM, Basting RT. Influence of in situ post bleaching times on resin composite shear bond strength to enamel and dentin. Am J Dent 2009; 22(6): 387-92.

[33] Gomes Torres CR, Fuzuko Koga A

The effects of anti-oxidant agents as neutralizers of bleaching agents on enamel bond strength. Braz J Oral Sci 2006, 5, 16: 971-76

[34] Carr AC, Tijerina T, Frei B. Vitamin C protects against and reverse specific hypochlorous acid- and chloraminesdependent modifications of low-density lipoprotein. Biochem J 2000; 2: 491-99.

[35] VanDuijn MM, Tijssen K, VanSteveninck J. Erythrocytes reduce extracellular ascorbate free radicals using intracellular ascorbate as an electron donor. $J$ Biol Chem 2000; 275: 2720-5.

[36] Moosavi H, Maleknejad F, Hoseinipour Z, Hatami L, Zeynali M. Antioxidant agents and their effects on shear bond strength of bleached enamel. J Contemp Dent Pract 2013 14(5): 871-5.

[37] Tabatabaei MH, Sakineh Arami S. Antioxidant effect on the shear bond strength of composite to bleached bovine dentin. Braz J Oral Sci 2011; 10(1); 33-36. 
[38] Bhusari CP, Sharma DS. Pattern of hydroxyapatite crystal growth on bleached enamel following the application of two antioxidants: an atomic force microscope study. J Clin Pediatr Dent 2017; 41(1): 38-47.

[39] Thandalam MT, Nesamani R, Kittappa K, Sanjeev K, Sekar M. Effect of various antioxidants on the shear bond strength of composite resin to bleached enamel: An in vitro study. J Conserv Dent 2014; 17(1): 22-26.

[40] Kadiyala A, Devalla S, Maroli S, Jayaprakash T. Effect of different anti-oxidants on shear bond strength of composite resins to bleached human enamel. J Clin Diag Res 2015; 9(11): 40-43.

[41] Martin JMH, Almeeida Jb, Rosa EAR. Effect of fluoride therapies on the surface roughness of human enamel exposed to bleaching agents. Quint Int 2010; 41: 71-78.

[42] Da Costa JB, Mazur RF. Effects of new formulas of bleaching gel and fluoride application on enamel microhardness: an in vitro study. Oper Dent 2007; 32(6): 589-94.

[43] Tschoppe P, Neumann K, Mueller J, Kielbassa AM. Effect of fluoridated bleaching gels on the remineralization of predemineralized bovine enamel in vitro. J Dent 2009; 37(2): 156-62.

[44] Niat AB, Yazdi FM, Koohestanian N. Effects of drying agents on bond strength of etch-and-rinse adhesive systems to enamel immediately after bleaching. J Adhes Dent 2012; 14(6): 511-6.

[45] Bradley G, Yao X, Ganguly A. Grape seed proanthocyanidins increase collagen biodegradation resistance in the dentin/ adhesive interface when included in an adhesive. J Dent 2010; 38: 908-15.

[46] Feiz M, Khoroushi M, Gheisarifar G. Bond strength of composite resin to bleached dentin: effect of using antioxidant versus buffering agent. J Dent 2011; 8(2); 61-64.

[47] Dias A, De Freitas H, Netto NG. Evaluation of the bond strength between a composite resin and enamel submitted to bleaching treatment and etched with Er:YAG laser. Photomed Laser Surg 2011; 29(2): 91-95.

[48] Torres C, Caneppele TM, Del Moral de Lazari R, Ribeiro CF, Borges AB. Effect of dental surface treatment with Nd:YAG and Er:YAG lasers on bond strength of resin composite to recently bleached enamel. Lasers Med Sci 2012; 27(4): 755-60.

[49] Basir MM, Bagher Rezvani MB, Chiniforush N, Moradi Z. Effect of $\mathrm{CO} 2$, Nd:YAG and Er:YAG lasers on microtensile bond strength of composite to bleachedenamel. Open Dent J 2016;10: 148-157.

[50] Li Q, Yu H, Wang Y. Color and surface analysis of carbamide peroxide bleaching effects on the dental restorative materials in situ. J Dent 2009; 37: 348-56.

[51] Jessica EM, Denys R, Annie JSG. Examen des effets du blanchiment externe sur les matériaux de restauration. J Can Dent Assoc 2011 77; 3: 164-67.

[52] Polydorou O, Hellwig E, Auschill TM. The effect of at-home bleaching on the microhardness of six esthetic restorative materials. J Am Dent Assoc 2007; 138(7): 978-84.

[53] Wendel A. Glutathione peroxidase. Enzymatic basis of detoxification. New York Amer Press 2009; 1: 333-53. 